\section{Discrete-Time Level-Crossing Analysis of Soft Handoff Performance in Cellular Networks}

Alexe E. Leu, Member, IEEE, and Brian L. Mark, Member, IEEE

\begin{abstract}
In this correspondence, we develop a formal method for solving a class of level-crossing problems in discrete-time with application to the analysis of soft handoff performance in cellular networks such as code-division multiple-access (CDMA) systems. In such networks, proper dimensioning of soft handoff parameters is critical to overcoming propagation impairments and providing a transparent radio access service for multiple user profiles. We obtain exact expressions for the cell assignment and active set update probabilities of a mobile station traveling along an arbitrary straight-line trajectory. We develop recursive algorithms to compute performance measures such as outage probability, macrodiversity gain, and signaling load. The discrete-time level-crossing analysis yields an accurate and efficient computational tool for designing and dimensioning high performance soft handoff algorithms while avoiding the need for approximations or time-consuming computer simulations.
\end{abstract}

Index Terms-Cellular networks, code-division multiple access (CDMA), level-crossing, macrodiversity, network dimensioning, performance analysis, recursive computation, soft handoff.

\section{INTRODUCTION}

In CDMA cellular networks such as IS-95, a mobile station (MS) maintains connectivity to an active set of base stations as it moves along a trajectory. The characteristics of CDMA spread spectrum communications permit the mobile station to receive transmissions from more than one base station simultaneously. This capability makes it possible to process a handoff from one base station to another within the active set of base stations maintained by the MS without any perceptible disturbance in communications for both voice and data traffic. Such a handoff procedure is known as soft handoff (cf. [2]-[5]). By contrast, cellular systems based on FDMA and/or TDMA, such as analog cellular or GSM, employ the more traditional hard handoff, whereby the mobile station maintains connectivity to at most one traffic channel at all times. In hard handoff, the mobile releases its current traffic channel and establishes connectivity via the new traffic channel.

To enable soft handoff in CDMA systems, signaling and data transmissions from base stations in the active set of the mobile station must be combined at a common point. The common point could be anywhere in the network, but typically it is located in the mobile switching center (MSC), which manages the radio link between the base stations and the mobile station. In IS-95, a decision is made at the common point to establish communications between the mobile station and one of the base stations in the active set when the signal from the selected base station becomes considerably stronger than the signals from the others. In the interim periods, the mobile station engages in simultaneous communications with all base stations in the active set.

Manuscript received December 9, 2002; revised December 14, 2005. This work was supported in part by the National Science Foundation under Grant 0133390 and a grant from the TRW Foundation. The material in this correspondence was presented in part at the IEEE International Conference on Communication (ICC), 2002.

A. E. Leu is with the Shared Spectrum Company, Vienna, VA 22182 USA (e-mail: aleu@ sharedspectrum.com).

B. L. Mark is with George Mason University, Fairfax, VA 22030 USA (e-mail: bmark@gmu.edu).

Communicated by L. Tassiulas, Associate Editor for Communication Networks.

Digital Object Identifier 10.1109/TIT.2006.876357
Compared to hard handoff schemes, soft handoff can increase the cell coverage area, or equivalently, lower the outage probability (cf. [6]). When the active set contains more than one base station, the mobile station provides diversity by combining the associated traffic channels in the forward or reverse links. Macrodiversity gain arises because less transmit power is required on the forward and reverse links, resulting in a reduction in the total system interference [6], [7]. Consequently, a further benefit of soft handoff is the longer battery life that results from the reduction in transmit power.

In this correspondence we present a discrete-time approach to evaluate soft handoff performance in cellular networks. ${ }^{1}$ The formalisms developed in the course of our analysis are more generally applicable to the numerical solution of a class of level-crossing problems (cf. [10]) in discrete-time. We derive an expression for the probability, as a function of the discrete-time parameter $k$, that a given base station will be assigned to the active set of a given mobile station. We also develop efficient recursive procedures for the exact evaluation of the cell assignment and active set update probabilities. Based on these soft handoff probabilities, a number of other important performance measures can be computed, which can be used to determine the average signaling load and macrodiversity gain for soft handoff. By taking into account the mobile station's trajectory in the network, a more accurate assessment of the outage probability can be obtained using the discrete-time approach compared with analyses that assume a stationary MS (cf. [6]).

Most of the earlier work on handoff analysis has relied primarily on computer simulation (cf. [11]-[13]). Vijayan and Holtzman [14] were among the first to develop an analytical model for hard handoff algorithms. Their model applied asymptotic results from the theory of level crossings [10] by approximating the sequences of handoffs to each of the two candidate base stations as two independent Poisson processes. The Poisson approximation is asymptotically true as the hysteresis threshold increases to infinity, but loses accuracy for smaller hysteresis values. Zhang and Holtzman [15], [16] proposed an alternative approach to analyze handoff based on the Gaussian properties of the sampled and processed received signal strengths. Their approach incorporated absolute signal strength thresholds in the handoff algorithm. Approximations for the handoff probabilities in [15] rely on some simplifying assumptions that can lead to inaccurate results. In [17], [18] simple closed-form approximations for the mean number of handoffs and mean handoff delay, respectively, for an MS moving between two base stations, are developed by curve-fitting to simulation results. In [19]-[21], asymptotic level-crossing results are used to derive approximations for the outage probability.

The correspondence is organized as follows. Section II describes the basic discrete-time signal propagation model for analyzing the performance of soft handoff algorithms. Section III develops a discrete-time characterization of soft handoff and derives the basic soft handoff performance metrics. Section IV develops the computational algorithms for efficiently evaluating the soft handoff probabilities. Section $\mathrm{V}$ presents numerical results illustrating the computation of soft handoff performance metrics using the discrete-time analysis. Finally, the correspondence is concluded in Section VI.

\section{Signal Propagation Model}

The cellular network is partitioned into a set of cells, where the $i$ th cell has an associated base station $B S_{i}$, located geographically by a position vector, $\boldsymbol{b}_{i}$, extended from a fixed origin. For convenience, we shall assume that the mobile station moves at a constant speed of $v$

\footnotetext{
${ }^{1}$ An early version of this work was presented in part in [1]. A discrete-time analysis of hard handoff performance is treated in [8], [9].
} 


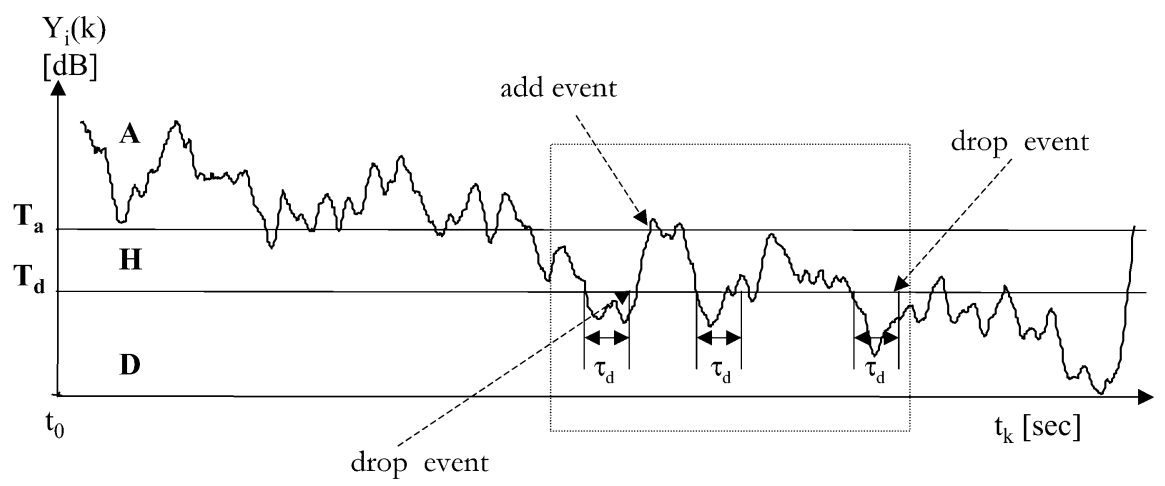

Fig. 1. Soft handoff sample path.

along a straight line from point $\boldsymbol{a}$ to point $\boldsymbol{b}$ in the cellular network. The position, $\boldsymbol{r}(t)$, of the mobile station at time $t$ is then given as follows:

$$
\boldsymbol{r}(t)=\boldsymbol{a}+v t \boldsymbol{e}, \quad 0 \leq t \leq T
$$

where $\boldsymbol{e}$ is a unit vector in the direction of the vector $\boldsymbol{b}-\boldsymbol{a}$.

\section{A. Received Signal Strength}

The pilot signal strength (in $\mathrm{dB}$ ), received at the mobile from base station $B S_{i}$ is given by (cf. [15], [16])

$$
Y_{i}(t)=m_{i}(t)+W_{i}(t)+Z_{i}(t)
$$

where

$$
m_{i}(t)=\kappa_{1}-\kappa_{2} \log \left(\left\|\boldsymbol{r}(t)-\boldsymbol{b}_{i}(t)\right\|\right)
$$

and $\left\{W_{i}(t)\right\}$ is an independent set of zero mean stationary Gaussian processes. The term $m_{i}(t)$ accounts for path loss, while $W_{i}(t)$ models the effect of lognormal (shadow) fading. The constants $\kappa_{1}$ and $\kappa_{2}$ depend on the mobile environment, with $\kappa_{1}=0 \mathrm{~dB}$ and $\kappa_{2}=30 \mathrm{~dB}$ being typical values in an urban setting. The autocorrelation function of $W_{i}(t)$ has been experimentally observed by Gudmundson [22] to have the following form:

$$
R_{W_{i}}(\tau)=\sigma_{W_{i}}^{2} \exp \left(-\frac{v|\tau|}{d_{0}}\right)
$$

where $\sigma_{W_{i}}$ is the standard deviation of the shadowing signal strength and the constant $d_{0}$ is called the decay factor. The process $Z_{i}(t)$ represents the effect of fast fading on the received pilot signal strength. The fast fading component can be effectively eliminated using the local averaging technique presented in [23], without appreciably modifying the characteristics of the shadowing and the path loss components. Therefore, we shall ignore the fast fading component in our subsequent analysis.

In practice, the mobile station samples the pilot signal strengths at discrete time instants $t_{k}=k \tau_{s}$, where $\tau_{s}$ is the sampling interval and $k$ ranges from 0 to $K$, where $K=\left\lfloor T / \tau_{s}\right\rfloor$. The discrete-time counterpart of (1) is given by:

$$
Y_{i}[k]=m_{i}[k]+W_{i}[k]
$$

where $Y_{i}[k]=Y\left(t_{k}\right), m_{i}[k]=m\left(t_{k}\right)$, and $W_{i}[k]=W\left(t_{k}\right)$. The processes $\left\{W_{i}[k]\right\}$ are independent, zero-mean, stationary Gaussian processes with autocorrelation function given by

$$
R_{W_{i}}(m)=\sigma_{W_{i}}^{2} \exp \left(\frac{-|m| d_{s}}{d_{0}}\right)
$$

where $d_{s}=v \tau_{s}$ is the distance between adjacent samples. From (3), one sees that the marginal density of $Y_{i}[k]$, which we denote by $f_{k}(y)$, is Gaussian with mean $m_{i}[k]$ and variance $\sigma_{W_{i}}^{2}$.

The shadow fading process $\left\{W_{i}[k]\right\}$ can be represented as a first-order autoregressive (AR) process by the following difference equation:

$$
W_{i}[k]=a W_{i}[k-1]+V_{i}[k]
$$

where $\left\{V_{i}[k]\right\}$ is a zero-mean, stationary white Gaussian noise process with variance $\sigma_{V_{i}}^{2}$. The parameters $\left(a, \sigma_{V_{i}}^{2}\right)$ of the AR model determine an autocorrelation function for $\left\{W_{i}[k]\right\}$ of the form

$$
R_{W_{i}}(m)=\sigma_{V_{i}}^{2} \frac{a^{|m|}}{1-a^{2}} .
$$

By comparing (6) and (4), the AR parameters for shadow fading are determined as follows:

$$
a=\exp \left(-\frac{d_{s}}{d_{o}}\right), \quad \sigma_{V_{i}}^{2}=\left(1-a^{2}\right) \sigma_{W_{i}}^{2} .
$$

From (3) and (5), one obtains a first-order difference equation in the received signal strength $Y_{i}[k]$ :

$$
Y_{i}[k]=a Y_{i}[k-1]+m_{i}[k]-a m_{i}[k-1]+V_{i}[k]
$$

which clearly shows that $\left\{Y_{i}[k]\right\}$ is a first-order AR process. For a mobile station moving along an arbitrary straight-line trajectory, sample paths of the received signal strength, $Y_{i}[k]$, can be generated for the purpose of Monte Carlo simulation (7). Later in the paper, we will make use of the first-order conditional density of $Y_{i}[k]$ given $Y_{i}[k-1]=x$, which we shall denote as $f_{k}(y \mid x)$. From (7), it is easy to see that $f_{k}(y \mid x)$ is a Gaussian density with mean $a x+m_{i}[k]-a m_{i}[k-1]$ and variance $\sigma_{V_{i}}^{2}$.

\section{B. Soft Handoff Model}

The assignment of cell $i$ to the mobile station's active set is determined by the time-evolution of the received signal strength process $\left\{Y_{i}(t)\right\}$. In IS-95 CDMA networks, cell $i$ is added to the active set of the MS when the value of $Y_{i}(t)$ exceeds the add threshold $T_{a}$. Cell $i$ is removed from the active set when $Y_{i}(t)$ falls below the drop threshold $T_{d}$ for an interval of at least $\tau_{d}$ seconds. In the discrete-time framework, the cell assignment decision is based on the process $\left\{Y_{i}[k]\right\}$. Cell $i$ is dropped from the active set when the value of $Y_{i}[k]$ is less than $T_{d}$ for $M$ consecutive discrete-time instants, where $M=\left\lceil\tau_{d} / \tau_{s}\right\rceil$. The parameters $T_{a}, T_{d}$, and $M$ determine the soft handoff algorithm. In general, these parameters may also depend on the cell $i$. Fig. 1 shows a sample path of the sampled pilot signal strength $\left\{Y_{i}[k]\right\}$ received from base station $B S_{i}$. 


\section{SOFT HANDOFF ANALYSIS}

Soft handoff behavior with respect to a fixed base station $B S_{i}$ can be characterized in terms of events induced by the process $\left\{Y_{i}[k]\right\}$. Partition the real line into three disjoint intervals defined as follows:

$$
A \triangleq\left[T_{a}, \infty\right), \quad H \triangleq\left(T_{d}, T_{a}\right), \quad D \triangleq\left(-\infty, T_{d}\right]
$$

where $A$ is the assignment region, $H$ is the hysteresis region, and $D$ is the drop region. The soft handoff behavior with respect to $B S_{i}$ can be couched in terms of these three regions. Denote the active (or assignment) set of the mobile station at time $k$ by $\mathcal{A}_{k}$. Let $Y_{i}[k \backslash r]$ denote the vector consisting of the $r$ most recent values of $\left\{Y_{i}[k]\right\}$ up to time $k$

$$
Y_{i}[k \backslash r] \triangleq\left(Y_{i}[k-r+1], \ldots, Y_{i}[k]\right) \text {. }
$$

The soft handoff rule at time $k \geq 1$ can be expressed as follows: If cell $i$ is not in the active set at time $k-1$, it enters the active set $\mathcal{A}_{k}$ at time $k$ if and only if $Y_{i}[k] \in A$. Conversely, if cell $i$ is in the active set at time $k-1$, it leaves the active set $\mathcal{A}_{k}$ at time $k$ if and only if $Y_{i}[k \backslash M] \in D^{M}$. We assume that cell $i$ is not in the active set at time $k=0$.

It is convenient to characterize the received signal strength in terms of the regions $A, H$, and $D$. Specifically, the received signal $Y_{i}[k]$ can be mapped to a symbol $S_{i}[k]$ defined on the alphabet $\mathcal{S}=\{A, H, D\}$ as follows:

$$
S_{i}[k] \triangleq \begin{cases}A, & \text { if } Y_{i}[k] \in A \\ H, & \text { if } Y_{i}[k] \in H \\ D, & \text { if } Y_{i}[k] \in D\end{cases}
$$

For $L \in \mathcal{S}$, let $L^{k}$ denote the string $L \ldots L$ ( $k$ times), when $k \geq 1$ and let $L^{0}$ denote the null string. In this context, a string of length $n \geq 1$ is equivalent to an $n$-tuple over the alphabet $\mathcal{S}$. The length of a string $s$ will be denoted by $|s|$. Let $\mathcal{S}^{k}$ denote the set of strings of length $k$, with $S^{0}$ denoting the empty set. The assignment of $B S_{i}$ at time $k$ is determined by the string $S_{i}[k \backslash k]$ that corresponds to the sample path $Y_{i}[k \backslash k]$. The soft handoff rule can be re-formulated in terms of the process $\{S[k]\}$. Thus, the set of all possible handoff behaviors of $B S_{i}$ can be characterized in terms of the set $\mathcal{S}^{+}$of all finite length strings over the alphabet $\mathcal{S}$, i.e., $\mathcal{S}^{+}=\cup_{k=1}^{\infty} \mathcal{S}^{k}$.

\section{A. Assignment Probabilities}

The assignment of $B S_{i}$ at time $k$ can be characterized in terms of subsets of the set $\mathcal{S}^{k}$. Let $E_{i}[k]$ denote the event that cell $i$ is assigned to the active set at time $k$. We assume that at time $k=0$, cell $i$ is not assigned to the active set. For $k \geq 1$, the cell assignment event $E_{i}[k]$ occurs if and only if $S_{i}[l]=A$ for some $l, 1 \leq l \leq k$ and $S_{i}[m \backslash M] \neq D^{M}$ for all $m, l \leq m \leq k$. In other words, $B S_{i}$ is assigned to the active set at time $k$ if the received signal strength falls in region $A$ at some time $l$ and after this time it never falls in region $D$ for $M$ consecutive time slots, at least up to time $k$.

Let $\mathcal{B}_{k}$ denote the set of strings of length $k$ over the alphabet $\mathcal{S}$ that do not contain the symbol $A$. One may express the set $\mathcal{B}_{k}$ as $\mathcal{B}_{k}=$ $\{H, D\}^{k}$. Define

$$
\mathcal{G}_{k} \triangleq \mathcal{B}_{k} \cap\left\{H, D H, D^{2} H, \ldots, D^{M-1} H\right\}^{+} .
$$

Note that $\mathcal{G}_{k}$ is the set of strings of length $k$ that end in the symbol $H$ and that do not contain the substring $D^{M}$ or the symbol $A$. The following lemma expresses the assignment event $E_{i}[k]$ as a disjoint union of more elementary events. ${ }^{2}$

\footnotetext{
${ }^{2}$ When sets $A$ and $B$ are disjoint we denote their union by $A \sqcup B$.
}

Lemma 1:

$$
E_{i}[k]=\bigsqcup_{l=0}^{M-1} \bigsqcup_{r=l+1}^{k}\left\{S_{i}[k \backslash r] \in A \mathcal{G}_{r-l-1} D^{l}\right\} .
$$

For a string $s \in \mathcal{S}^{+}$, of length $|s| \leq k$, we introduce the following notation:

$$
p_{k, i}(s) \triangleq P\left\{Y_{i}[k \backslash|s|] \in s\right\}=P\left\{S_{i}[k \backslash|s|]=s\right\} .
$$

Thus, $p_{k, i}(s)$ is the probability that the last $|s|$ values of the sequence $Y_{i}[k \backslash k]$ fall in the regions defined by the string $s$. The probability that cell $i$ is assigned to the active set is denoted by $P_{i}[k]=P\left\{E_{i}[k]\right\}$. Similarly, the probability that cell $i$ is not assigned to the active set is denoted by $\bar{P}_{i}[k]=P\left\{\bar{E}_{i}[k]\right\}$. We have that $P_{i}[k]+\bar{P}_{i}[k]=1$ for all $k \geq 0$. In particular, we have assumed that $\bar{P}_{i}[0]=1$. Given the representation of the event $E_{i}[k]$ in Lemma 1 , an expression for the assignment probability follows immediately.

Proposition 1:

$$
P_{i}[k]=\sum_{l=0}^{M-1} \sum_{r=l+1}^{k} \sum_{s \in A \mathcal{G}_{r-l-1} D^{l}} p_{k, i}(s) .
$$

Equation (11) provides a closed-form expression for the assignment probability but is not convenient for computation. An efficient procedure for computing $P_{i}[k]$ will be developed in Section IV.

\section{B. Active Set Update Events}

One of the most critical issues in the dimensioning, design and optimization of mobile networks is the modeling of the signaling required to maintain the active set of the mobile stations. An update of the active set occurs when a base station is either dropped or added to the active set. Such an event corresponds roughly to the concept of a handoff between two base stations in hard handoff algorithms. The main difference is that the active set may contain two or more base stations at any given time (cf. [8]).

An important performance measure for the soft handoff problems is the mean number of updates of the active set required by the mobile while moving along a particular trajectory. Let $A_{i}[k]$ denote the event that base station $B S_{i}$ is added to the active set of the mobile station at time $k$. The event $A_{i}[k]$ can be expressed as follows:

$$
A_{i}[k]=\bar{E}_{i}[k-1] \cap E_{i}[k] .
$$

Similarly, we define $D_{i}[k]$ as the event that $B S_{i}$ is dropped from the active set at time $k$. The event $D_{i}[k]$, can be expressed as follows:

$$
D_{i}[k]=E_{i}[k-1] \cap \bar{E}_{i}[k] .
$$

The following lemma expresses the add and drop events as unions of disjoint events.

Lemma 2:

$$
\begin{gathered}
A_{i}[k]=\left\{S_{i}[k \backslash k] \in \mathcal{G}_{k-1} A\right\} \sqcup \\
\bigsqcup_{r=1}^{M-1}\left\{S_{i}[k \backslash k] \in D^{r} \mathcal{G}_{k-r-1} A\right\} \sqcup \\
\bigsqcup_{r=M}^{k}\left\{S_{i}[k \backslash r] \in D^{M} \mathcal{G}_{r-M-1} A\right\} \\
D_{i}[k]=\bigsqcup_{r=M+1}^{k}\left\{S_{i}[k \backslash r] \in A \mathcal{G}_{r-M-1} D^{M}\right\} .
\end{gathered}
$$

Let $P_{A_{i}}[k]=P\left(A_{i}[k]\right)$ denote the probability that an add event occurs at time $k$. Similarly, let $P_{D_{i}}[k]=P\left(D_{i}[k]\right)$ denote the drop prob- 
ability at time $k$. Expressions for the add and drop probabilities follow immediately from Lemma 2. Efficient numerical procedures for computing these probabilities will be developed in Section IV.

\section{Derived Performance Metrics}

From the cell assignment probabilities, a number of handoff performance measures can be derived. The mean size of the active set or macrodiversity degree at a given instance $k$ is given by

$$
n_{k}=\sum_{i \in \mathcal{N}_{k}} P_{i}[k]
$$

The mean size of the active set over the entire trajectory is therefore given by

$$
\bar{N}=\frac{1}{K} \sum_{k=1}^{K} n_{k}
$$

The probability that the active set contains a given number of base stations can be expressed as follows:

$$
P\left\{\left|\mathcal{A}_{k}\right|=n\right\}=\sum_{\mathcal{S} \subseteq \mathcal{N}_{k},|\mathcal{S}|=n} \prod_{i \in \mathcal{S}} P_{i}[k] \prod_{j \notin \mathcal{A}_{k}} \bar{P}_{j}[k]
$$

where $\mathcal{N}_{k}$ is the set of neighbor cells at time $k$. The outage probability ${ }^{3}$ is the probability that the active set is empty and is given by:

$$
P_{o}[k]=P\left\{\left|\mathcal{A}_{k}\right|=0\right\} .
$$

Hence, the mean number of handoffs or update events over the entire trajectory is given by

$$
\bar{N}_{u}=\frac{1}{K} \sum_{k=1}^{K} \sum_{i \in \mathcal{A}_{k}}\left(P_{A_{i}}[k]+P_{D_{i}}[k]\right)=\frac{1}{K} \sum_{k=1}^{K} \nu[k] .
$$

The above performance metrics can be used to evaluate CDMA system capacity (cf. [24], [25]), while capturing the effect of mobility on soft handoff behavior.

\section{COMPUTATIONAL AlgORITHMS}

The closed-form expressions for soft handoff probabilities derived in Section III do not readily lend themselves to computation. In this section, we develop efficient algorithms for computing the soft handoff probabilities.

\section{A. Non-Assignment Probability}

We shall characterize the set of strings that correspond to the nonassignment event $\bar{E}_{i}[k]$. We recursively define the following sequences of sets of strings:

$$
\mathcal{S}_{k, H D^{l}} \triangleq \mathcal{S}_{k-1, H D^{l-1}} \cdot D
$$

${ }^{3}$ We assume that the received pilots from base stations are independent of each other. where $1 \leq l \leq M-1$ and $k \geq 2$

$$
\mathcal{S}_{1, H} \triangleq\{H\} \text { and } \mathcal{S}_{1, H D^{m}}=\emptyset
$$

and $1 \leq m \leq M-1$. To complete the recursive definition, we define

$$
\mathcal{S}_{k, D} \triangleq \begin{cases}\left\{D^{k}\right\} \sqcup \bigsqcup_{m=1}^{k-1} \mathcal{S}_{k, H D^{m}}, & 1 \leq k \leq M \\ \mathcal{S}^{k-M} D^{M} \sqcup \bigsqcup_{m=1}^{M-1} \mathcal{S}_{k, H D^{m}}, & k>M\end{cases}
$$

and

$$
\mathcal{S}_{k, H} \triangleq \mathcal{S}_{k-1, D} \cdot H \cup \mathcal{S}_{k-1, H} \cdot H
$$

The following result, which can be proved by induction, establishes that

1) $\mathcal{S}_{k, H D^{m}}(0 \leq m \leq M-1)$ is the set of all strings of length $k$ that end in the substring $H D^{m}$ and for which nonassignment occurs at time $k$.

2) $\mathcal{S}_{k, D}$ is the set of all strings of length $k$ that end in $D$ and for which nonassignment occurs at time $k$.

Proposition 2: For $k \geq 1$ and $0 \leq m \leq M-1$, the following hold:

$$
\bar{E}_{i}[k] \cap\left\{S_{i}[k \backslash m+1]=H D^{m}\right\}=\left\{S_{i}[k \backslash k] \in \mathcal{S}_{k, H D m}\right\}
$$

and

$$
\bar{E}_{i}[k] \cap\left\{S_{i}[k]=D\right\}=\left\{S_{i}[k \backslash k] \in \mathcal{S}_{k, D}\right\}
$$

The set of strings corresponding to the nonassignment event $\bar{E}_{i}[k]$ at time $k$ can be expressed as a disjoint union of sets as follows:

$$
S_{k} \triangleq S_{k, H} \sqcup S_{k, D}
$$

The next corollary suggests a recursive computational algorithm for computing the nonassignment probabilities. The proof follows immediately from Proposition 2.

Corollary 1:

$$
\bar{E}_{i}[k]=\left\{S_{i}[k \backslash k] \in S_{k}\right\} .
$$

We now introduce a family of operators defined on the space of functions $\Psi: \mathcal{R} \rightarrow \mathcal{R}^{+}$as follows:

$$
\begin{aligned}
& \mathbf{A}_{k} \Psi(y) \triangleq \int_{A} \Psi(x) f_{k}(y \mid x) d x \\
& \mathbf{D}_{k} \Psi(y) \triangleq \int_{D} \Psi(x) f_{k}(y \mid x) d x \\
& \mathbf{H}_{k} \Psi(y) \triangleq \int_{H} \Psi(x) f_{k}(y \mid x) d x .
\end{aligned}
$$

Here, $f_{k}(y \mid x)$ denotes the conditional density of $Y[k]$ given that $Y[k-$ $1]=x$, which is Gaussian with mean $a x+m_{i}[k]-a m_{i}[k-1]$ and variance $\sigma_{V_{i}}^{2}$ (cf. (7)). Next, we define a family of $M-1$ sequences of functions

$$
h_{k, D^{m}}: \mathcal{R} \rightarrow \mathcal{R}^{+}, \quad k \geq 1, \quad 0 \leq m \leq M-1
$$

where $\mathcal{R}$ denotes the real line and we abbreviate $h_{k, D^{0}}$ as $h_{k}$.

$$
\begin{aligned}
h_{k}(y) & \triangleq f_{k}(y), \\
h_{k, D^{m}}(y) & \triangleq \mathbf{D}_{k} h_{k-1, D^{m-1}}(y)
\end{aligned}
$$


with $1 \leq m \leq \min (M-1, k)$ and $h_{1, D^{m}}(y) \triangleq 0$. Here, $f_{k}(x)$ denotes the marginal (Gaussian) density of $Y[k]$ (cf. (3)). An expression for the probability of a drop event at time $k$ is given as follows.

Proposition 3:

$$
p_{k}\left(D^{M}\right)=P\left\{S_{i}[k \backslash k] \in \mathcal{S}^{k-M} D^{M}\right\}=\int_{D} h_{k, D} D^{M-1}(y) d y
$$

for $k \geq M$

Next, we introduce a sequence of functions $g_{k, H D^{m}}: \mathcal{R} \rightarrow \mathcal{R}^{+}$, $m=0, \ldots, M-2$. For $k \geq 2$ define $q_{k}: \mathcal{R} \rightarrow \mathcal{R}^{+}$as follows:

$$
q_{k}(y) \triangleq h_{k, D^{\min (M, k)-1}}(y)+\sum_{m=1}^{\min (M, k)-2} g_{k, H D^{m}}(y)
$$

For $k \geq 1$ define

$$
\begin{aligned}
1 \text { define } & \triangleq \int_{k}(y) \\
g_{k, H}(y) & \triangleq \mathbf{H}_{k-1}(x) f_{k-1}(y \mid x) d x+g_{k, H}(y) \\
g_{k, H D^{m}}(y) & \triangleq \mathbf{D}_{k} g_{k-1, H D^{m-1}}(y)
\end{aligned}
$$

with $1 \leq m<\min (M-1, k)$ and

$$
g_{1, H D^{m}}(y) \triangleq 0, \quad 0 \leq m<M-1 .
$$

Using the operator notation, the definition (24) may be written as

$$
g_{k}(y) \triangleq \mathbf{D}_{k} q_{k-1}(y)+\mathbf{H}_{k} g_{k-1}(y)
$$

The following expression for $g_{k, H D^{m}}(y)$ can be derived by induction.

Lemma 3:

$$
g_{k, H D^{m}}(y)=\sum_{s \in \mathcal{S}_{k-1, H D^{m-1}}} \int_{s} f_{1, \ldots, k}\left(\boldsymbol{y}_{k-1}, y\right) d \boldsymbol{y}
$$

where $0 \leq m \leq M-2, \boldsymbol{y} \triangleq\left(y_{1}, \ldots, y_{k-1}\right)$ and $d \boldsymbol{y} \triangleq d y_{1} \cdots d y_{k-1}$.

The following result is an immediate consequence of Lemma 3.

Corollary 2:

$$
P\left\{S[k \backslash k] \in \mathcal{S}_{k, H D^{m}}\right\}=\int_{D} g_{k, H D^{m-1}}(y) d y,
$$

for $1 \leq m \leq M-1$.

The next corollary follows from definitions (16) and (23), Proposition 3, and Corollary 2.

Corollary 3:

$$
P\left\{S[k \backslash k] \in \mathcal{S}_{k, D}\right\}=\int_{D} q_{k}(y) d y .
$$

Similar to Lemma 3, the following lemma can be proved by induction on $k$.

Lemma 4:

$$
g_{k}(y)=\sum_{s \in \mathcal{S}_{k-1}} \int_{s} f_{1, \ldots, k}\left(y_{1}, \ldots, y_{k-1}, y\right) d y_{1} \ldots d y_{k-1}
$$

The next corollary follows immediately from Lemma 4.
Corollary 4:

$$
P\left\{S[k \backslash k] \in \mathcal{S}_{k, H}\right\}=\int_{H} g_{k}(y) d y .
$$

The cell nonassignment probabilities can then be expressed as follows.

Theorem 1:

$$
\bar{P}_{i}[k]=\int_{D} q_{k}(y) d y+\int_{H} g_{k}(y) d y .
$$

Proof: The result follows directly from (19) and Corollaries 1, 3, and 4 .

\section{B. Update Probabilities}

In this section, we develop a recursive procedure for evaluating the update probabilities. The add and drop events can be expressed in terms of the assignment and nonassignment events as follows.

Proposition 4:

$$
\begin{aligned}
& A_{i}[k]=\bar{E}_{i}[k-1] \cap\left\{S_{i}[k] \in A\right\} \\
& D_{i}[k]=E_{i}[k-1] \cap\left\{S_{i}[k \backslash M] \in D^{M}\right\} .
\end{aligned}
$$

The probability of an add event can be expressed in terms of the functions defined above for computing the assignment probability.

Theorem 2:

$$
P_{A_{i}}[k]=\int_{A} g_{k}(y) d y
$$

Proof: The result follows from Proposition 4, Corollary 1, and Lemma 4.

To compute the probability of a drop event, we must consider the set of strings resulting in assignment at time $k-1$ and ending in the substring $D^{M}$. To that end, we define the following sets of strings (for $k \geq 1)$ :

$$
\begin{aligned}
\mathcal{F}_{k, D^{l}} & \triangleq \mathcal{F}_{k-1, D^{l-1}}, \quad l \geq 1 \\
\mathcal{F}_{k} & \triangleq \tilde{\mathcal{S}}_{k-1} \cdot H \sqcup \mathcal{S}^{k-1} \cdot A, \quad k \geq 1 \\
\tilde{\mathcal{S}}_{k} & \triangleq \bigsqcup_{m=0}^{\min (k, M)-1} \mathcal{F}_{k, D^{m}, \quad k \geq 1}
\end{aligned}
$$

with $\tilde{\mathcal{S}}_{0} \triangleq \emptyset$. The following proposition implies that $\mathcal{F}_{k, D^{m}}(0 \leq$ $m \leq M-1)$ is the set of strings that result in nonassignment at time $k$ and that end in the substring $D^{m}$.

Proposition 5:

$$
E_{i}[k] \cap\left\{S_{i}[k \backslash m]=D^{m}\right\}=\left\{S_{i}[k \backslash k] \in \mathcal{F}_{k, D^{m}}\right\}
$$

for $1 \leq m \leq M-1$.

The next corollary expresses the drop event in terms of the set $\mathcal{F}_{k, D^{M}}$.

Corollary 5: 


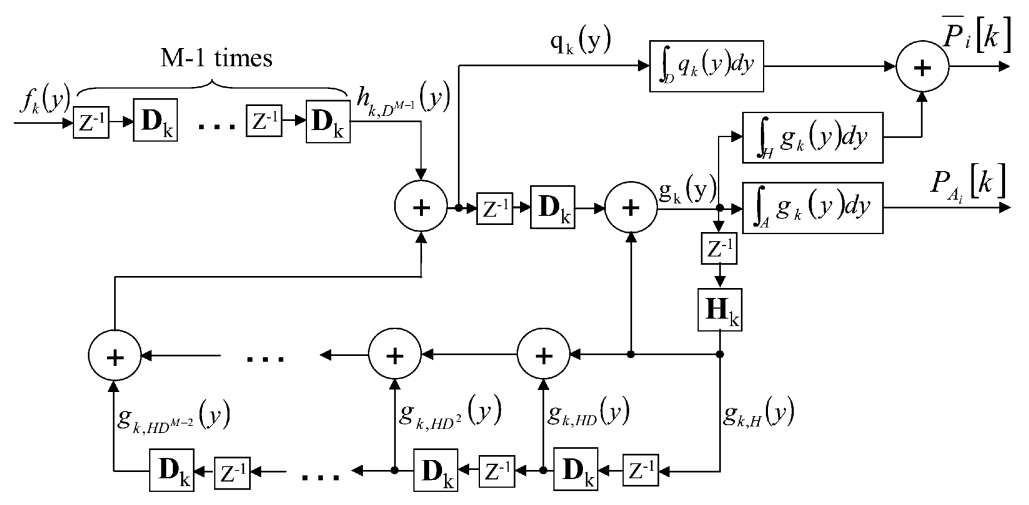

Fig. 2. Recursive computational scheme for assignment and add probabilities.

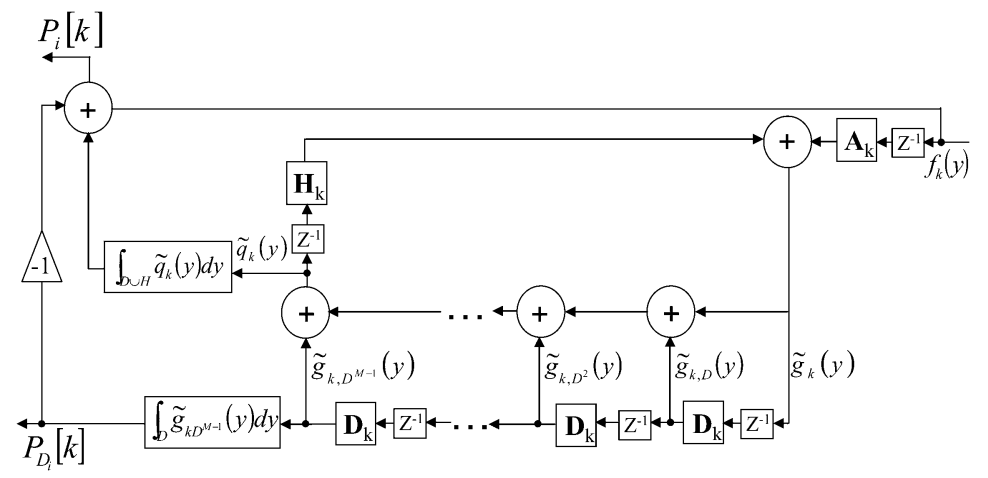

Fig. 3. Recursive computational scheme for drop probabilities.

To compute the drop probabilities, we introduce the following sequence of functions:

$$
\begin{aligned}
\tilde{q}_{k}(y) & \triangleq \sum_{m=0}^{\min (k, M)-1} \tilde{g}_{k, D^{m}}(y), \quad k \geq 1 \\
\tilde{g}_{k}(y) & \triangleq \mathbf{H}_{k} \tilde{q}_{k-1}(y)+\mathbf{A}_{k} f_{k-1}(y) \\
\tilde{g}_{k, D^{l}}(y) & \triangleq \mathbf{D}_{k} \tilde{g}_{k-1, D^{l-1}}(y)
\end{aligned}
$$

for $1 \leq l \leq M-1, k \geq 1$, and $\tilde{g}_{1, D^{m}}(y) \triangleq 0$ for all $y \in \mathcal{R}$ and $0 \leq m \leq M-1$. The next lemma can be proved by induction on $k$.

Lemma 5:

$$
\begin{aligned}
\tilde{q}_{k}(y) & =\sum_{s \in \tilde{\mathcal{S}}_{k-1}} \int_{s} f_{1, \ldots, k}\left(\boldsymbol{y}_{k-1}, y\right) d \boldsymbol{y}_{k-1} \\
\tilde{g}_{k, D^{m}}(y) & =\sum_{s \in \mathcal{F}_{k-1, D^{m}}} \int_{s} f_{1, \ldots, k}\left(\boldsymbol{y}_{k-1}, y\right) d \boldsymbol{y}_{k-1}
\end{aligned}
$$

for $0 \leq m \leq k-1$.

Finally, we arrive at the next theorem, which gives an expression for the drop probabilities. The proof of the theorem follows straightforwardly from Lemma 5 and Corollary 5.

Theorem 3:

$$
P_{D_{i}}[k]=\int_{D} \tilde{g}_{k, D}{ }^{M-1}(y) d y, \quad k \geq M+1 .
$$

The assignment probabilities can also be computed directly in terms of the functions $\tilde{q}_{k}(y)$ as follows.

Theorem 4:

$$
P_{i}[k]=\int_{D \cup H} \tilde{q}_{k}(y) d y-\int_{D} \tilde{g}_{k, D^{M-1}}(y) d y+\int_{A} f_{k}(y) d y
$$

for $k \geq 1$.

\section{Efficient Implementation Schemes}

Fig. 2 depicts an efficient recursive implementation scheme of Theorems 1 and 2 for computing the nonassignment and add event probabilities, respectively. The symbol $z^{-1}$ indicates a delay of one sampling interval between the input and output points. Each delay block of $z^{-1}$ is followed by one of the three operators $\mathbf{A}, \mathbf{D}, \mathbf{H}$, or a specified integration operation. From Fig. 2, we see that computation of the add event probability requires $2 M$ integration operations, where $M$ is the number of time steps in the drop timer interval. Computation of the nonassignment probability requires $2 M+1$ integrations. Fig. 3 depicts an implementation of Theorems 3 and 4 for computing the drop event and assignment probabilities, respectively. From Fig. 3, we see that the computation of both the assignment and drop probabilities requires $M+3$ integration operations. Therefore, computation of the assignment, add event, and drop event probabilities at step $k$ requires a total of $3 M+3$ integrations.

\section{NUMERICAL RESUlTS}

The problem of dimensioning the handoff algorithm is to determine an optimal set of values for the handoff parameters $T_{a}, T_{d}$, and $M$ according to given performance criteria. In practice, handoff performance metrics must be evaluated over a large range of values for the handoff parameters in order to dimension the cellular network properly. We present some representative results of applying the discrete-time analysis to obtain the handoff performance metrics of interest. Fig. 4 illustrates the cellular network structure that is used for the soft handoff performance evaluation. For our numerical computations, we set the distance between two adjacent base stations to $2000 \mathrm{~m}$ and the main system parameters as follows: $\kappa_{1}=0 \mathrm{~dB}, \kappa_{2}=30 \mathrm{~dB}, \sigma_{W_{i}}=6 \mathrm{~dB}$, $d_{o}=20 \mathrm{~m}, d_{s}=1 \mathrm{~m}$. These values are typical for an urban environment (cf. [16]).

To validate the discrete-time analysis, we also show results obtained using computer simulation. In our numerical studies, computation of 


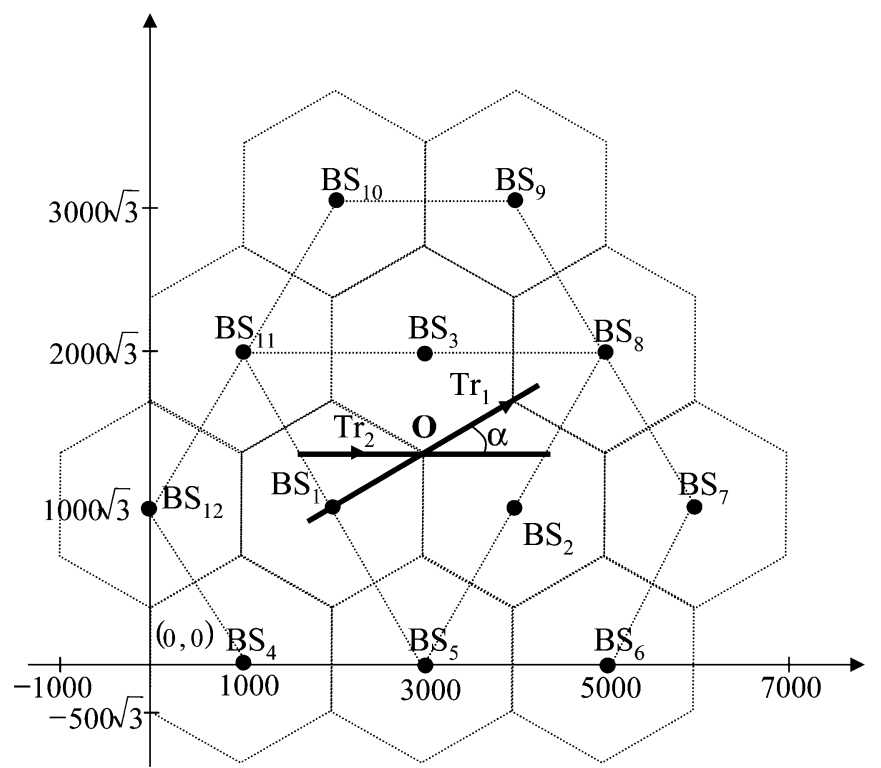

Fig. 4. Cellular network scenario for soft handoff.

the assignment probability via the recursive procedure was about two orders of magnitude faster than computer simulation for $95 \%$ confidence intervals. To optimize the simulation procedure, pre-computed sample paths were stored in a file, whereas the computational procedure does not require any additional storage. The gain in efficiency achieved by our computational method becomes particularly significant in multiple user, multiple service scenarios.

Within the triangle formed by $B S_{5}, B S_{8}$, and $B S_{11}$ two sample trajectories, $\operatorname{Tr}_{1}$ and $\operatorname{Tr}_{2}$ are shown. Both trajectories pass radially through the point $O$ at the intersection of cells 1,2 , and 3 . In the numerical results discussed below, we focus on trajectory $\operatorname{Tr}_{2}$. As the mobile station moves along $\operatorname{Tr}_{2}$, we consider seven particular base stations: $B S_{2}, B S_{3}, B S_{5}, B S_{6}, B S_{7}, B S_{8}$ and $B S_{9}$. Due to symmetry relative to trajectory $\operatorname{Tr}_{2}$, the assignment probabilities for $B S_{2}, B S_{6}$, $B S_{7}, B S_{8}$, and $B S_{9}$ are the same as the corresponding assignment probabilities for $B S_{1}, B S_{4}, B S_{12}, B S_{11}$, and $B S_{10}$, respectively.

Fig. 5 illustrates the assignment probabilities for the seven selected base stations as the mobile moves along trajectory $\operatorname{Tr}_{2}$. The parameters of the soft handoff algorithm are set as follows: drop threshold $T_{d}=-94 \mathrm{~dB}$, add threshold $T_{a}=-92$, and drop timer interval $M=2$. The dotted curves represent the assignment probabilities obtained from simulation along the trajectory $\operatorname{Tr}_{2}$, while the continuous curves obtained from our analytical model. Again, the analytical curves match closely with those from simulation. Observe that the curves for $B S_{3}$ and $B S_{5}$ are symmetrical about the halfway point, which can also be deduced from the geometrical symmetry in the cellular structure. The curve for $B S_{3}$ lies under the curve of $B S_{5}$ since the $B S_{3}$ is located at a greater distance than $B S_{5}$ from the trajectory $\operatorname{Tr}_{2}$. Initially, $B S_{8}, B S_{7}, B S_{6}$, and $B S_{9}$ are assigned to the active set with probabilities close to 0 , which then increase monotonically as the mobile approaches the ending point of the trajectory $\operatorname{Tr}_{2}$. The assignment probability curve of $B S_{2}$ starts with a value of approximately 0.1 and increases monotonically as the mobile reaches the ending point of $\operatorname{Tr}_{2}$.

Fig. 6 shows the update probabilities for the same set of base stations that is considered for the trajectory $\operatorname{Tr}_{2}$. The figure shows the results from simulation with the $95 \%$ confidence intervals shown explicitly. The curves from our analysis agree with the curves obtained

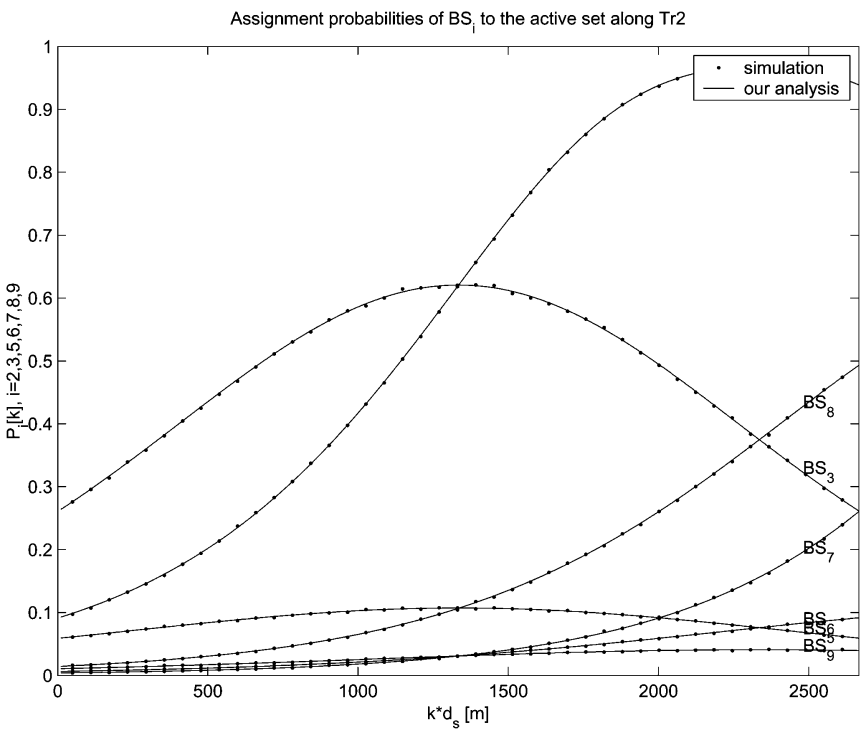

Fig. 5. Cell assignment probability along trajectory $\operatorname{Tr}_{2}$.

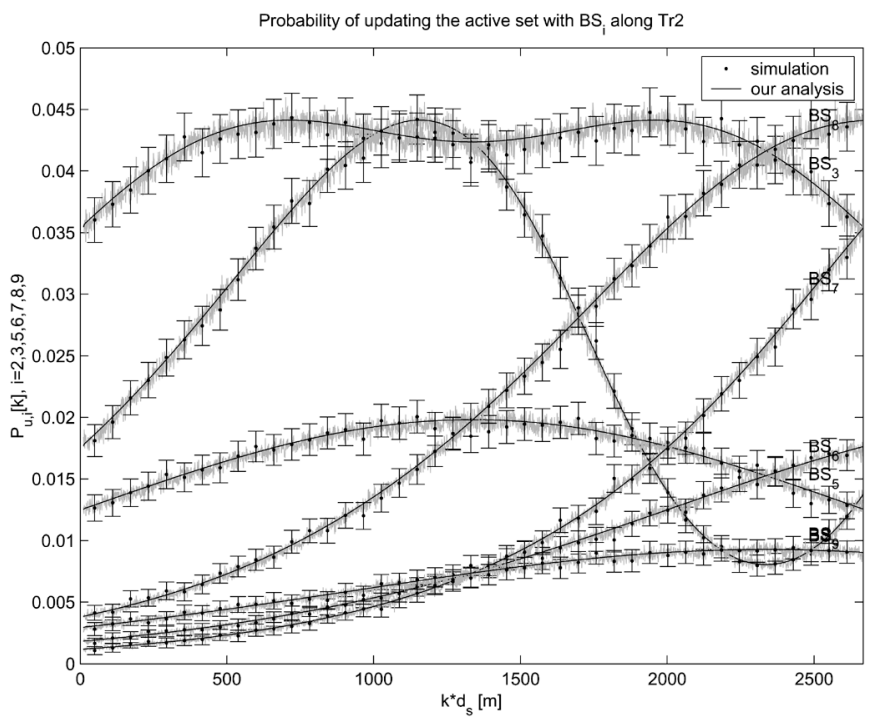

Fig. 6. Active set update probability along trajectory $\operatorname{Tr}_{2}$.

from simulation. As one would expect, the update probability curves for $B S_{3}$ and $B S_{5}$ are symmetrical about the halfway point. For $B S_{2}$, the curve increases, reaches a peak value around $1200 \mathrm{~m}$ and then decreases monotonically to a minimum value around $2350 \mathrm{~m}$ after which increases monotonically for the rest of the trajectory of about $350 \mathrm{~m}$. Along trajectory $\operatorname{Tr}_{2}$, the mobile moves closer to $B S_{6}, B S_{7}, B S_{8}$ and $B S_{9}$. Hence, the update probability curves for these base stations increase monotonically.

Figs. 7 and 8 show the assignment probability and update probability curves for $B S_{3}$ along trajectory $\operatorname{Tr}_{2}$ for different values of the drop timer interval $M$. In Fig. 7, one sees that the assignment probability curve increases as $M$ is increased. On the other hand, as seen in Fig. 8, the update probability decreases as $M$ increases. Thus, the signaling load can be decreased by increasing $M$, but doing so results in higher resource utilization due to a larger active set size. It is interesting to note that the rate of decrease of the update probability curve with increasing $M$ is higher than the rate of increase of the assignment probability 


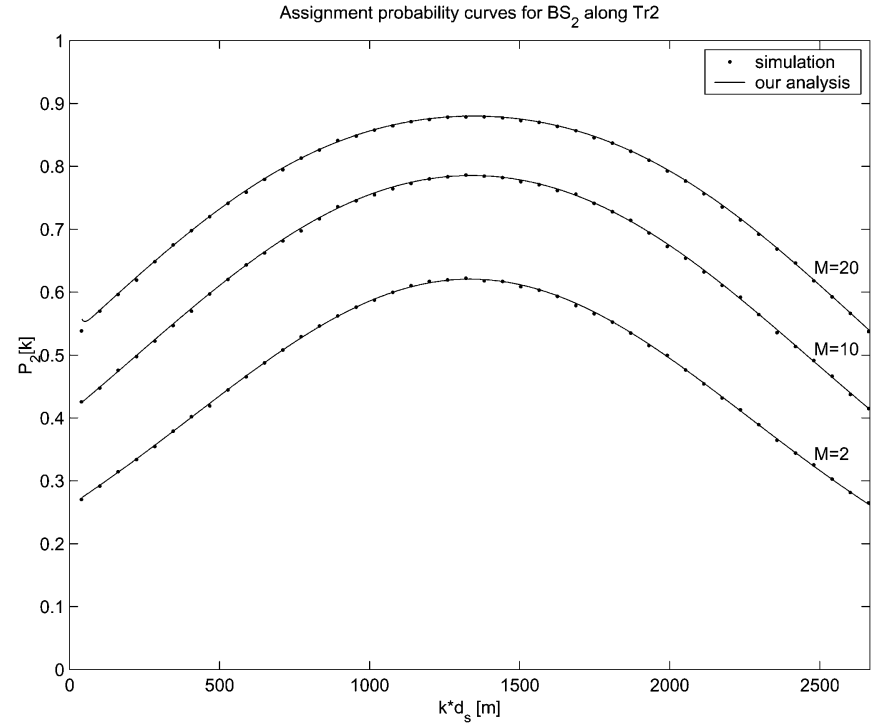

Fig. 7. Assignment probability for $B S_{2}$ along trajectory $\operatorname{Tr}_{2}$ for different values of the drop timer interval.

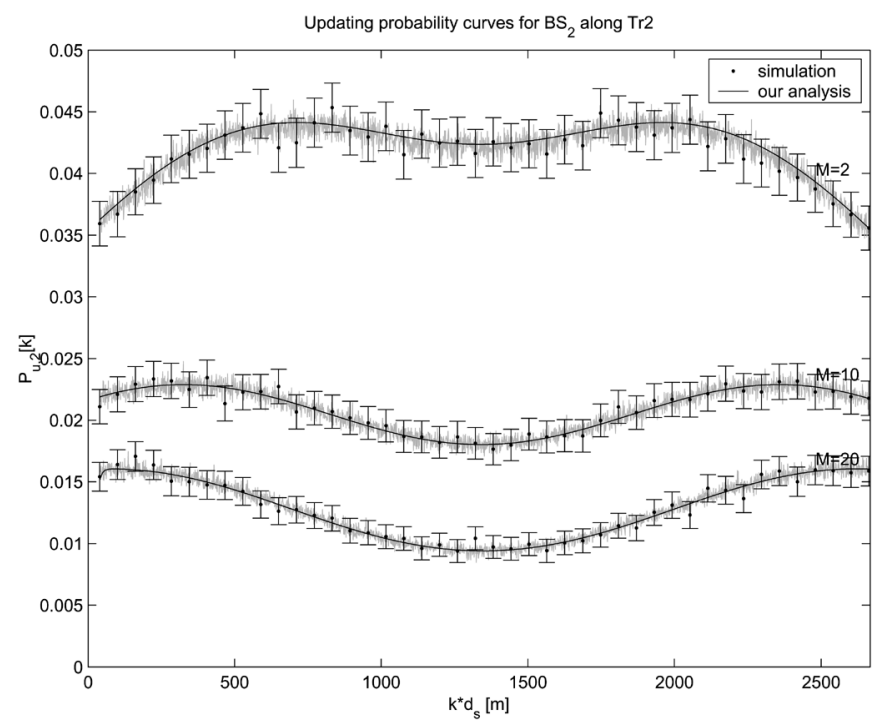

Fig. 8. Update probability for $B S_{2}$ along trajectory $\operatorname{Tr}_{2}$ for different values of the drop timer interval.

curve. This suggests the existence of an optimum operating point for the value of $M$ given a suitably defined cost function.

\section{CONCLUSION}

We presented a discrete-time level-crossing analysis of soft handoff performance for a mobile station travelling along a given trajectory in cellular networks. We developed recursive computational procedures to evaluate the cell assignment and update probabilities efficiently and accurately. The handoff analysis is based only on sampled pilot signal strength measurements received by the mobile station and is applicable to arbitrary mobile trajectories. Dimensioning the handoff algorithm is a complex and extremely time-consuming task, especially when using computer simulation. Our approach provides an efficient computational tool that can greatly simplify the task of dimensioning cellular networks, especially in multiple user/service scenarios. The physical layer handoff analysis presented here can also be incorporated into higher level simulation studies of overall network performance.
We have assumed that the handoff parameters are static as in the IS-95 standard, but the analysis can be extended to adaptive handoff algorithms that dynamically change the parameter values over time. Traditionally, level-crossing problems have been studied in the continuous-time domain with asymptotic approximations and relatively simple level-crossing rules (cf. [10]). The discrete-time formalism to analyze handoff performance can in principle be applied to a larger class of level-crossing problems.

\section{REFERENCES}

[1] A. E. Leu and B. L. Mark, "Discrete-time analysis of soft handoff in CDMA cellular networks," in Proc. IEEE Int. Conf. Commun. (ICC), New York, Apr./May 2002, pp. 3222-3226.

[2] G. P. Pollini, "Trends in handover design," IEEE Commun. Mag., vol. 34, no. 3, pp. 82-90, Mar. 1996.

[3] K. Pahlavan, "Handoff in hybrid mobile data networks," IEEE Pers. Commun., vol. 7, no. 2, pp. 34-37, Apr. 2000.

[4] N. D. Tripathi, J. H. Reed, and H. F. VanLandingham, "Handoff in cellular systems," IEEE Personal Communications, vol. 5, no. 6, pp. 26-37, Dec. 1998.

[5] D. Wong and T. J. Lim, "Trends in handover design," IEEE Pers. Commun., vol. 4, no. 6, pp. 6-16, Dec. 1997.

[6] A. J. Viterbi, A. M. Viterbi, K. S. Gilhousen, and E. Zehavi, "Soft handoff extends CDMA cell coverage and increases reverse link capacity," IEEE J. Sel. Areas Commun., vol. 12, pp. 1281-1287, Oct. 1994.

[7] G. L. Stüber, Principles of Mobile Communication, 2nd ed. New York: Kluwer Academic, 2002.

[8] A. E. Leu and B. L. Mark, "A discrete-time approach to analyze hard handoff performance in cellular networks," IEEE Trans. Wireless Commun., vol. 3, no. 5, pp. 1721-1733, Sep. 2004.

[9] _ _ "An efficient timer-based hard handoff algorithm for cellular networks," in Proc. IEEE Wireless Commun. Networking Conf. (WCNC), New Orleans, LA, Mar. 2003.

[10] I. F. Blake and W. C. Lindsey, "Level-crossing problems for random processes," IEEE Trans. Inf. Theory, vol. 19, pp. 295-315, May 1973.

[11] R. Beck and H. Panzer, "Strategies for handover and dynamic channel allocation in micro-cellular mobile radio systems," in Proc. IEEE Veh. Technol. Conf. (VTC), 1989, pp. 178-185.

[12] W. R. Mende, "On the hand-over rate in future cellular systems," in Proc. IEEE Veh. Technol. Conf. (VTC), 1988, pp. 358-361.

[13] B. Gudmundson and O. Grimlund, "Handoff in microcellular based personal telephone systems," in Proc. WINLAB Workshop, East Brunswick, NJ, Oct. 1990

[14] R. Vijayan and J. M. Holtzman, "A model for analyzing handoff algorithms," IEEE Trans. Veh. Technol., vol. 42, pp. 351-356, Aug. 1993.

[15] N. Zhang and J. M. Holtzman, "Analysis of handoff algorithms using both absolute and relative measurements," IEEE Trans. Veh. Technol., vol. 45, pp. 174-179, Feb. 1996.

[16] _ _ "Analysis of CDMA soft-handoff algorithm," IEEE Trans. Veh. Technol., vol. 47, pp. 710-714, May 1998.

[17] G. P. Pollini, "Handover rates in cellular systems: Toward a closed form approximation," in Proc. IEEE Globecom, 1997, vol. 2, pp. 711-715.

[18] S. Ulukus and G. P. Pollini, "Handover delay in cellular wireless systems," in Proc. IEEE Int. Conf. Commun., 1998, vol. 3, pp. 1370-1374.

[19] P. C. Chen, N. B. Mandayam, and J. M. Holtzman, "Minimum duration outage for cellular systems with reception diversity," in Proc. IEEE Int. Conf. Univ. Pers. Commun., 1996, vol. 1, pp. 245-249.

[20] J. Lai and N. B. Mandayam, "Minimum duration outages in Rayleigh fading channels," IEEE Trans. Commun., vol. 49, pp. 1755-1761, Oct. 2001.

[21] N. B. Mandayam, P. C. Chen, and J. M. Holtzman, "Minimum duration outage for cellular systems: A level crossing analysis," Wireless Pers. Commun., vol. 7, no. 2/3, pp. 135-146, 1998.

[22] M. Gudmundson, "Correlation model for shadowing fading in mobile radio systems," Electron. Lett., vol. 27, no. 23, pp. 2145-2146, Nov. 1991.

[23] A. E. Leu and B. L. Mark, "Modeling and analysis of fast handoff algorithms for microcellular networks," in Proc. IEEE/ACM Int. Symp. Model., Anal. Simul. Comput. Telecommun. Syst. (MASCOTS), Fort Worth, TX, Oct. 2002, pp. 321-328.

[24] R. P. Narrainen and F. Takawira, "Performance analysis of soft handoff in CDMA cellular networks," IEEE Trans. Veh. Technol., vol. 50, no. 6, pp. 1507-1517, Nov. 2001.

[25] C. Lee and R. Steele, "Effect of soft and softer handoffs on CDMA cellular capacity," IEEE Trans. Veh. Technol., vol. 47, pp. 830-841, Jul. 1998. 